\title{
Screening for cerebrotendinous xanthomatosis
}

\author{
James J. Pitt • Heidi Peters
}

Received: 1 July 2014 /Revised: 28 July 2014 / Accepted: 19 August 2014 / Published online: 19 September 2014

(C) SSIEM 2014

Dear Editors,

A recent paper in JIMD (Mignarri et al 2014) provided a useful summary of the clinical findings in cerebrotendinous xanthomatosis (CTX) and developed a clinical scoring system to prioritise suspected patients for further investigation with plasma cholestanol measurements. As the authors emphasise, early detection of CTX is important because treatment with chenodeoxycholic acid can prevent or ameliorate disease progression, including neurological deterioration. However, the approach advocated by Mignarri et al still requires the clinician to recognise CTX as a possible diagnosis. Early symptoms can be non-specific and the possibility of CTX may not be recognised by non-specialist clinicians.

Another approach to diagnosing CTX is the wider application of less targeted, biochemical screening. Newborn screening for CTX has been advocated but is still in the developmental phase and may have a low benefit:cost ratio due to the rarity of CTX and the cost of testing. An alternative is urine metabolite screening to detect CTX patients. Urine bile alcohols, such as cholestanepentol glucuronide (CPG), are grossly increased in CTX patients. CPG testing can be incorporated into a general urine metabolic screening panel of amino acids, acyl carnitines, organic acids and other metabolic markers analysed by electrospray tandem mass spectrometry (Pitt 2007). Screen positive samples can be further evaluated with a full bile alcohol glucuronide profile to detect other abnormal bile alcohols excreted in CTX.

This approach has been used to test all urine samples submitted for metabolic screening in the state of Victoria, Australia

Communicated by: Ronald J. A. Wanders

J. J. Pitt $(\bowtie) \cdot$ H. Peters

Murdoch Childrens Research Institute, Melbourne, VIC, Australia

e-mail: james.pitt@vegs.org.au (population 5.8 million) for CPG since 2004. Screening was easily incorporated into the existing panel of metabolites with minimal laboratory costs. Approximately 23,000 urine samples have been screened with the detection of four CTX patients and no false positive results. Three patients from two families were detected as adults with neurological symptoms and CTX testing was performed as part of their differential diagnosis. The fourth patient was diagnosed at age 3 years after urine metabolic screening consequent to chronic diarrhoea and developmental delay. Significantly, CTX was not suspected by the treating physician who requested screening.

Urine screening for CPG therefore provides a complementary approach to the standardised clinical evaluation advocated by Mignarri et al for diagnosing CTX. CTX patients with non-specific early symptoms such as jaundice, diarrhoea or epilepsy are likely to have urine metabolic screening performed even when CTX is clinically unsuspected and will thus be detected by this approach.

Yours faithfully,

James Pitt and Heidi Peters

Conflict of interest None declared.

Animal rights This article does not contain any studies with animal subjects performed by the any of the authors.

\section{References}

Mignarri A, Gallus GN, Dotti MT, Federico A (2014) A suspicion index for early diagnosis and treatment of cerebrotendinous xanthomatosis. J Inherit Metab Dis 37(3):421-9

Pitt JJ (2007) High-throughput urine screening for Smith-Lemli-Opitz syndrome and cerebrotendinous xanthomatosis using negative electrospray tandem mass spectrometry. Clin Chim Acta 380(1-2): 81-8 